\title{
Generic selection criteria for safety and patient benefit [VI]: Comparing the physicochemical and pharmaceutical properties of brand-name, generic, and OTC felbinac tapes
}

\author{
Yuko Wada ${ }^{1}$, Yukie Takaoka ${ }^{1}$, Mitsuru Nozawa ${ }^{2}$, Miho Goto ${ }^{2}$, Ken-ichi Shimokawa ${ }^{1}$, \\ Fumiyoshi Ishii ${ }^{1, *}$
}

${ }^{1}$ Department of Pharmaceutical Sciences, Meiji Pharmaceutical University, Tokyo, Japan;

${ }^{2}$ Triad Japan Co., Ltd., Kanagawa, Japan.

\begin{abstract}
Summary We measured the $\mathrm{pH}$, water-vapor permeability, adhesive force, peeling-force, elongation rate, support flexibility, and peeling time of medicinal and over-the-counter (OTC) tape preparations containing felbinac. When measuring the $\mathrm{pH}$ of each preparation, Felnabion (pH 4.5) was weakly acidic, and EMEC and Tokuon (pH 7.0) were neutral. When measuring the water-vapor permeability of each preparation, that of a generic product, EMEC $\left(380 \mathrm{~g} / \mathrm{m}^{2} / 24\right.$ $\mathrm{h}$ ), was twice as high as that of a brand-name product, Seltouch $\left(189 \mathrm{~g} / \mathrm{m}^{2} / 24 \mathrm{~h}\right)$. The adhesive force was measured using the ball tack test. The adhesive forces of OTC drugs, Salomethyl, Homepass, and Tokuhon $(1.04 \mathrm{~g})$, were higher than that of Seltouch $(0.06 \mathrm{~g})$. Concerning peeling-force measurement, the peeling-forces of a generic product, Falzy $(4.15 \mathrm{~N})$, and an OTC drug, Omuneed (4.89 N), were higher than that of Seltouch $(0.91 \mathrm{~N})$. The elongation rates of a generic product, Sumilu (319\%), and OTC drugs, Nabolin (298\%) and Homepass $(299 \%)$, were higher than that of Seltouch $(251 \%)$, but that of Tokuhon $(\mathbf{7 2 \%})$ was lower. The support flexibilities of EMEC (150 degrees) and Tokuhon (131 degrees) were higher than that of Seltouch (96 degrees). In addition, the peeling time of Seltouch was $120 \mathrm{~min}$ or more, whereas those of EMEC and Nabolin were 1.4 and $0.2 \mathrm{~min}$, respectively. These results suggest that the differences in pharmaceutical properties, such as the $\mathrm{pH}$, water-vapor permeability, adhesive force, peeling-force, elongation rate, support flexibility, and peeling time, among the preparations markedly influence patients' subjective comfortableness. The results of this study facilitated individuals' comfortableness-matched drug selection.
\end{abstract}

Keywords: Brand-name products, generic products, OTC, tape preparation, felbinac

\section{Introduction}

A topical preparation containing a nonsteroidal antiinflammatory drug, felbinac-containing tape preparation, is selected for many patients to relieve muscular or articular pain (1). Concerning this drug, a brand-name medicinal product, Seltouch, and many generic products

Released online in J-STAGE as advance publication October 4, 2016.

*Address correspondence to:

Dr. Fumiyoshi Ishii, Department of Pharmaceutical Sciences, Meiji Pharmaceutical University,

2-522-1, Noshio, Kiyose, Tokyo 204-8588, Japan.

E-mail: fishii@my-pharm.ac.jp are commercially available, and a large number of switched OTC drugs are also commercially available. The active component contents of these switched OTC drugs are equivalent to those of medicinal products; similar effects may be obtained. As for merit, the former can be purchased at a drug store without prescription. As anyone can purchase the switched OTC drugs without consulting a hospital, these drugs may reduce expanded health expenditures as an important social problem. However, the effects are not sufficient $(2,3)$.

In the future, to promote the widespread use of proprietary drugs, including switched OTC drugs, it is necessary to appeal to their wide usefulness. To provide adequate drug information to patients, pharmacists must understand the pharmaceutical properties of both 
Table 1. Products used in this experiment

\begin{tabular}{|c|c|c|c|c|c|}
\hline Product name & Abbreviated name & Class & Company & Size $(\mathrm{cm})$ & Serial number \\
\hline Seltouch $^{\circledR}$ tape 70 & Seltouch & brand-name & Teikoku Seiyaku Co., Ltd. & $10 \times 14$ & $12 \mathrm{~K} 06 \mathrm{~A}$ \\
\hline Felbinac $^{\circledR}$ tape 70 mg "EMEC" & EMEC & generic & Kyukyu Pharmaceutical Co., Ltd. & $10 \times 14$ & 2W21P \\
\hline Sumilu $^{\circledR}$ tape $70 \mathrm{mg}$ & Sumilu & generic & Mikasa Seiyaku Co., Ltd. & $10 \times 14$ & 301222 \\
\hline Flex $^{\circledR}$ tape $70 \mathrm{mg}$ & Flex & generic & Hisamitsu Pharmaceutical Co., Inc. & $10 \times 14$ & N301U \\
\hline Felnabion $^{\circledR}$ tape $70 \mathrm{mg}$ & Felnabion & generic & Okayama Taiho Pharmaceutical Co., Ltd. & $10 \times 14$ & $2 \mathrm{I} 89$ \\
\hline Falzy $^{\circledR}$ tape $70 \mathrm{mg}$ & Falzy & generic & Nipro Patch Co., Ltd. & $10 \times 14$ & FJ001 \\
\hline Nabolin $^{\circledR}$ Felbinac 70 & Nabolin & OTC & Kyukyu Pharmaceutical Co., Ltd. & $10 \times 14$ & $2 \mathrm{~T} 11 \mathrm{~W}$ \\
\hline Patecs ${ }^{\circledR}$ Felbinastar VL & Patecs & OTC & Daiichi Sankyo Healthcare Co., Ltd. & $10 \times 14$ & GK002 \\
\hline Faitus $^{(\mathbb{B})} 3.5 \alpha \mathrm{L}$ & Faitus & OTC & Hisamitsu Pharmaceutical Co., Inc. & $10 \times 14$ & $\mathrm{~N} 221 \mathrm{U}$ \\
\hline Salomethyl $^{\circledR}$ FB Patch 35 & Salomethyl & OTC & Toko Pharmaceutical Industrial Co., Ltd. & $7 \times 10$ & LXBK \\
\hline Omuneed $^{\mathbb{R}}$ FB Plaster & Omuneed & OTC & Teikoku Seiyaku Co., Ltd. & $7 \times 10$ & 6205 \\
\hline Homepass ${ }^{\circledR}$ FR & Homepass & OTC & Oishi Koseido Co., Ltd. & $7 \times 10$ & 241212 \\
\hline Tokuhon $^{\circledR}$ Felbina Plaster & Tokuhon & OTC & Tokuhon Co., Ltd. & $5.6 \times 6.5$ & $2 \mathrm{E} 02$ \\
\hline Menfla ${ }^{\circledR}$ Felbinac Onkan & Menfla & OTC & Kyoritsu Pharmaceutical Industres Co., Ltd. & $7 \times 10$ & 13E02 \\
\hline
\end{tabular}

OTC: over-the-counter drug.

medicinal and proprietary drugs. However, information on these drugs is not sufficient (4).

We have compared the pharmaceutical properties of various medicinal drugs between brand-name and generic products (5-9). In this study, with respect to felbinaccontaining tape preparations, which are used in many patients, we compared the pharmaceutical properties of 6 medicinal drugs ( 1 brand-name and 5 generic products) and 8 OTC drugs.

\section{Materials and Methods}

\subsection{Materials}

As felbinac tape, a brand-name product, Seltouch ${ }^{\circledR}$ tape 70 (Teikoku Seiyaku Co., Ltd., Kagawa, Japan), generic products, Felbinac ${ }^{\circledR}$ tape $70 \mathrm{mg}$ "EMEC" (Kyukyu Pharmaceutical Co., Ltd., Tokyo, Japan), Sumilu ${ }^{\circledR}$ tape $70 \mathrm{mg}$ (Mikasa Seiyaku Co., Ltd., Tokyo, Japan), Flex $^{\circledR}$ tape 70 mg (Hisamitsu Pharmaceutical Co., Inc., Osaka, Japan), Felnabion ${ }^{\circledR}$ tape $70 \mathrm{mg}$ (Okayama Taiho Pharmaceutical Co., Ltd., Okayama, Japan), Falzy ${ }^{\circledR}$ tape 70 mg (Nipro Patch Co., Ltd., Saitama, Japan), and OTC products, Nabolin ${ }^{\circledR}$ Felbinac 70 (Kyukyu Pharmaceutical Co., Ltd., Tokyo, Japan), Patecs ${ }^{\circledR}$ Felbinastar VL (Daiichi Sankyo Healthcare Co., Ltd., Tokyo, Japan), Faitus ${ }^{\circledR}$ $3.5 \alpha \mathrm{L}$ (Hisamitsu Pharmaceutical Co., Inc., Osaka, Japan), Salomethyl ${ }^{\mathbb{E}}$ FB Patch 35 (Toko Pharmaceutical Industrial Co., Ltd., Tokyo, Japan), Omuneed ${ }^{\mathbb{B}}$ FB Plaster (Teikoku Seiyaku Co., Ltd., Kagawa, Japan), Homepass ${ }^{\circledR}$ FR (Oishi Koseido Co., Ltd., Saga, Japan), Tokuhon ${ }^{\circledR}$ Felbina Plaster (Tokuhon Co., Ltd., Tokyo, Japan), Menfla ${ }^{\circledR}$ Felbinac Onkan (Kyoritsu Pharmaceutical Industres Co., Ltd., Tokyo, Japan), were purchased and used in this experiment (Table 1). All the other reagents were of analytical grade.

\subsection{Measurement of $p H$}

We measured $\mathrm{pH}$ values, as described by Wada et al.
(8). Briefly, each preparation was cut into sections measuring $70 \times 50 \mathrm{~mm}$, placed in sample bottles containing $20 \mathrm{~mL}$ of purified water, and agitated for 24 h. Subsequently, the $\mathrm{pH}$ of the solution was measured using a Benchtop pH meter F-74 (HORIBA, Ltd., Kyoto, Japan). For each product, measurement was conducted 3 times, and the mean was adopted as its $\mathrm{pH}$ value.

\subsection{Measurement of the water-vapor permeability}

The water-vapor permeability test was performed, as described by Hiyoshi et al. (10). Briefly, $10 \mathrm{~mL}$ of purified water was placed in a glass container, and its opening was covered with a round section of each product measuring $40 \mathrm{~mm}$ in diameter. After the periphery was fixed with a piece of elastic paraffin film (Parafilm ${ }^{\circledR}$ : Bemis Flexible Packaging, IL, USA), the weight was measured. Subsequently, each sample was allowed to stand for $24 \mathrm{~h}$ in an environment chamber KCL-2000W (Tokyo Rikakikai Co., Ltd., Tokyo, Japan) under the following conditions: temperature, $40^{\circ} \mathrm{C}$; relative humidity, $50 \%$. Additionally, the weight was measured. The water-vapor permeability was calculated from the rate of change in the weight using the following formula: water-vapor permeability $(\mathrm{g} /$ $\left.\mathrm{m}^{2} / 24 \mathrm{~h}\right)=\left(\mathrm{W}_{0}-\mathrm{W}_{1}\right) \times 10,000 / \mathrm{A}\left[\mathrm{W}_{0}\right.$ : weight before testing $(\mathrm{g}), \mathrm{W}_{1}$ : weight after testing $(\mathrm{g}), \mathrm{A}$ : area of the glass container's opening $\left(\mathrm{cm}^{2}\right)$ ]. With respect to each product, a measurement was conducted 3 times, and the mean was regarded as the water-vapor permeability.

\subsection{Measurement of the adhesive force}

The adhesive force was measured according to the inclined ball tack test method established in the Japanese Industrial Standards (JIS) (11). A section of each product measuring $10 \times 200 \mathrm{~mm}$ was longitudinally attached on the inclined ball tuck examination device TransTack ${ }^{\circledR}$ Ball-Tack Meter (CosMED Pharmaceutical 
Co. Ltd., Kyoto, Japan) under the following conditions: temperature, $25^{\circ} \mathrm{C}$; relative humidity, $50 \%$. The weight of the maximum ball number was measured by the inclined ball tuck examination device. With respect to each product, a measurement was conducted 3 times, and the mean was regarded as the adhesive force.

\subsection{Measurement of the peeling-force}

The peeling-force was measured according to the adhesive tape/sheet test method described by Miura et al. (12). Briefly, a slide table P90-200N (Imada Co., Ltd., Aichi, Japan) for 90 degrees peeling test was fixed on an MX2-500N (Imada Co., Ltd., Aichi, Japan) stand for measurements, and controlled caliper ethylene vinyl acetate (EVA) membrane (3M CoTran ${ }^{\mathrm{TM}}$ 9702, 3M Japan Ltd., Tokyo Japan) as a type of artificial skin was fixed on the slide table. On its surface, a section of each product measuring $30 \times 52 \mathrm{~mm}$ was longitudinally attached. In addition, a cylindrical weight (4 kg) was rolled over each product. Subsequently, the peeling-force was measured by pinching a 2-mm area of the upper margin with a film clip FC-40 (Imada Co., Ltd., Aichi, Japan $)$ and pulling it at a constant rate $(1 \mathrm{~mm} / \mathrm{sec})$ so that the adhesive surface was vertical to a digital force gauge, ZP-50N (Imada Co., Ltd., Aichi, Japan), until the tape had been completely exfoliated from the EVA membrane. With respect to each product, a measurement was conducted 3 times, and the mean was regarded as the peeling-force.

\subsection{Measurement of the elongatedness}

An elongatedness test was performed, as described by Terada et al. (13). The end $(20 \mathrm{~mm})$ of a section of each product measuring $20 \times 100 \mathrm{~mm}$ was fixed on an experimental table with the adhesive surface. The maximum extension distance $(\mathrm{cm})$ was measured by pulling the other side. The elongatedness (\%) was calculated from the values before and after. For each product, a measurement was conducted 3 times, and the mean was regarded as the elongatedness.

\subsection{Measurement of the support flexibility}

Each preparation was cut into a square $(50 \times 50 \mathrm{~mm})$ and half areas $(25 \times 50 \mathrm{~mm})$ were stuck on the edge of the test table, and the bending angle of the remaining half was measured. The flexibility of the support was evaluated. For each product, a measurement was conducted 3 times, and the mean was regarded as the flexibility.

\subsection{Measurement of time before exfoliating from skin when the preparation was wet with water}

Each preparation was cut by into a square $(50 \times 50 \mathrm{~mm})$ and affixed by constant pressure on artificial skin (bioskin
No.10A; Beaulax Co., Ltd., Saitama, Japan), and stirred in purified water on a magnetic stirrer $(1,000 \mathrm{rpm})$. The peeling time for each preparation was measured. For each product, a measurement was conducted 3 times, and the mean was regarded as the peeling time.

\subsection{Statistical analysis}

The values were compared using Dunnett's test. A $p$-value of 0.05 or 0.01 was regarded as significant.

\section{Results}

\subsection{Measurement of $p H$}

The $\mathrm{pH}$ is an important factor reflecting the stability of the active component in each preparation or skin irritability. The results of $\mathrm{pH}$ measurements for each product are shown in Figure 1. There were marked differences in $\mathrm{pH}$ among the brand-name, generic, and OTC products. The $\mathrm{pH}$ values of generic products, Sumilu (pH 4.7), Flex (pH 4.7), Felnabion ( $\mathrm{pH} 4.5$ ), and Falzy ( $\mathrm{pH} 4.6$ ), were similar to that of a brand-name product, Seltouch ( $\mathrm{pH}$ 4.6). Those of OTC products, Patecs (pH 4.8), Faitus ( $\mathrm{pH} 4.9$ ), Salomethyl (pH 4.7), Omuneed ( $\mathrm{pH} 4.6$ ), and Menfla ( $\mathrm{pH} 4.7)$, were also similar to that of Seltouch. On the other hand, the $\mathrm{pH}$ values of a generic product, EMEC ( $\mathrm{pH} 7.0)$, and OTC products, Nabolin ( $\mathrm{pH}$ 5.6), Homepass ( $\mathrm{pH} 5.1$ ), and Tokuhon ( $\mathrm{pH} 7.0)$, were higher. Significance tests of these products were conducted. There were significant differences between EMEC/Nabolin/Homepass/Tokuhon and Seltouch $(p<0.01)$ (Figure 1).

\subsection{Measurement of the water-vapor permeability}

The water-vapor permeability of each preparation may induce maceration stimuli when the skin water permeability is low on attachment. We measured the water-vapor permeability of each product. The results are shown in Figure 2. There were marked differences

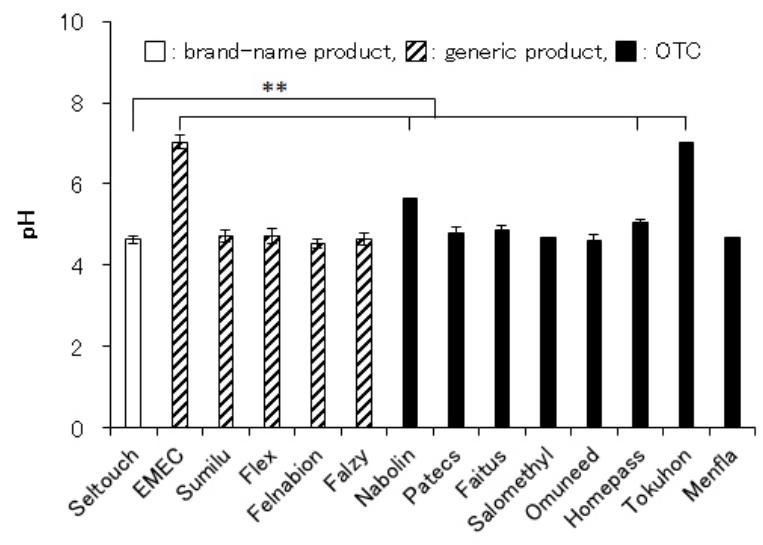

Figure 1. $\mathbf{p H}$ comparison of various products $(\boldsymbol{n}=\mathbf{3}) . * * p$ $<0.01$ (brand-name vs. generics, Dunnett's test). 
in the water-vapor permeability among the products; the water-vapor permeabilities of EMEC $\left(380 \mathrm{~g} / \mathrm{m}^{2} / 24\right.$ h), Sumilu (318 g/m $/ 24 \mathrm{~h})$, and Nabolin $\left(269 \mathrm{~g} / \mathrm{m}^{2} / 24\right.$ h) were higher than that of Seltouch $\left(189 \mathrm{~g} / \mathrm{m}^{2} / 24 \mathrm{~h}\right)$. In addition, the results showed significant differences between EMEC, Sumilu, Nabolin and Seltouch $(p<$ $0.01, p<0.01$, and $p<0.05$, respectively) (Figure 2).

\subsection{Measurement of the adhesive force}

Preparations with a strong adhesive force may not peel off when attached to the skin, whereas those with a weak adhesive force tend to peel off. We measured the adhesive force of each product. The results are shown in Figure 3. There were marked differences in the adhesive force among the products; the adhesive forces of OTC products, Salomethyl, Homepass, and Tokuhon (1.04 g), were higher than that of Seltouch $(0.06 \mathrm{~g})$. In addition, the results showed significant differences between all generic/OTC products and Seltouch $(p<0.01)$ (Figure 3 ).

\subsection{Measurement of the peeling-force}

The peeling-force refers to a force required to peel off the

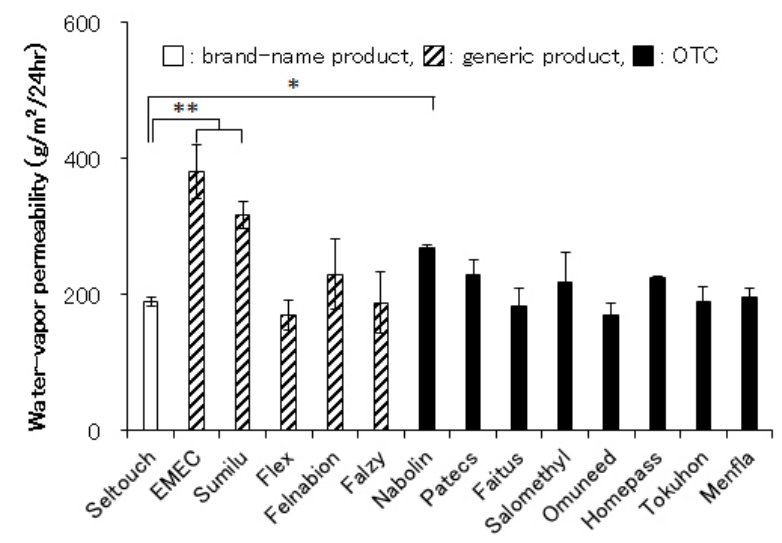

Figure 2. Comparison of water-vapor permeability of the various products $(\boldsymbol{n}=\mathbf{3}) . * p<0.05, * * p<0.01$ (brand-name $v s$. generics, Dunnett's test).

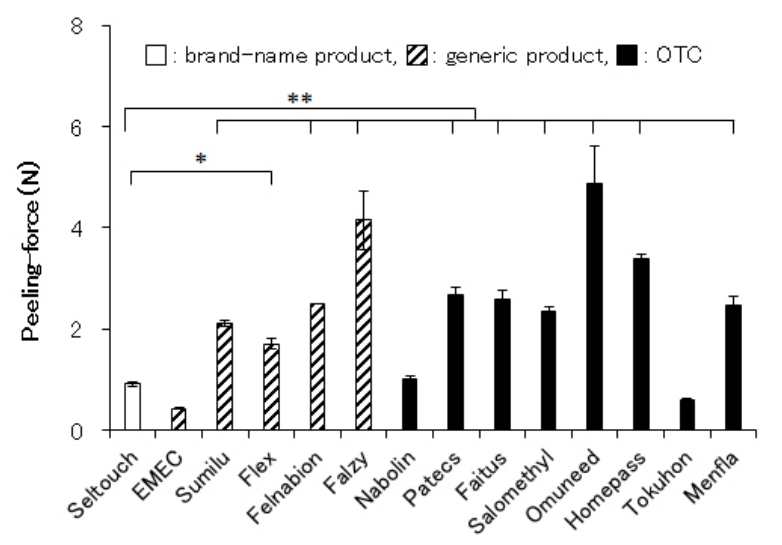

Figure 4. Comparison of peeling-force of the various products $(\boldsymbol{n}=\mathbf{3}) .{ }^{*} p<0.05,{ }^{*} p<0.01$ (brand-name $v s$. generics, Dunnett's test). preparation after attachment. We measured the peelingforce of each product. The results are shown in Figure 4. The peeling-forces of Falzy (4.15 N), Omuneed (4.89 $\mathrm{N})$, and Homepass $(3.39 \mathrm{~N})$ were higher than that of Seltouch $(0.91 \mathrm{~N})$, whereas those of EMEC $(0.42 \mathrm{~N})$ and Tokuhon $(0.58 \mathrm{~N})$ were lower than that of Seltouch. In addition, significance tests of various preparations were conducted. Generic products, Sumilu, Felnabion, and Falzy, and OTC products, Patecs, Faitus, Salomethyl, Omuneed, Homepass, and Menfla, showed significant differences in comparison with Seltouch $(p<0.01)$. There was also a significant difference between Flex and Seltouch $(p<0.05)$ (Figure 4).

\subsection{Measurement of the elongation rate}

In many products, a stretchy material is used for the support layer to prevent turning-up or peeling after attachment to articular regions such as the knees and elbows. The results of elongation-rate measurement are shown in Figure 5. The elongation rates of Sumilu (319\%), Flex (282\%), Nabolin (298\%), Faitus (287\%), and Homepass (299\%) were higher than that of Seltouch $(251 \%)$. On the other hand, the elongation rate of

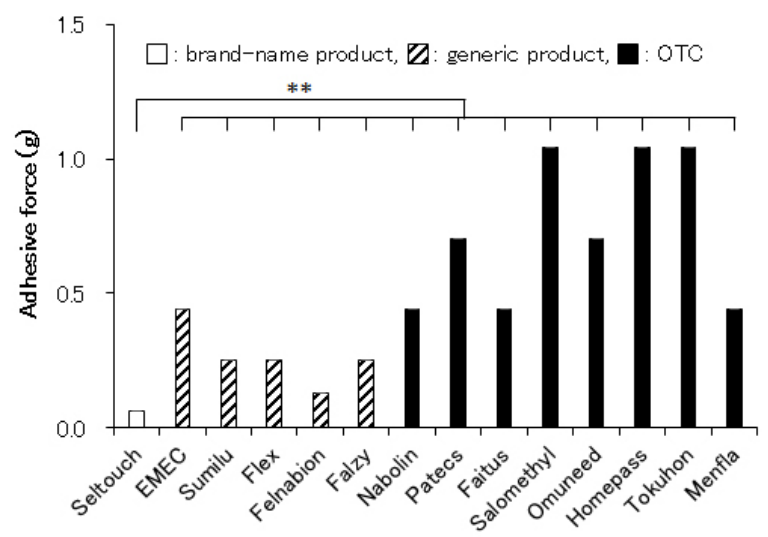

Figure 3. Comparison of adhesive force of the various products $(\boldsymbol{n}=\mathbf{3}) .{ }^{* *} p<0.01$ (brand-name $v s$. generics, Dunnett's test).

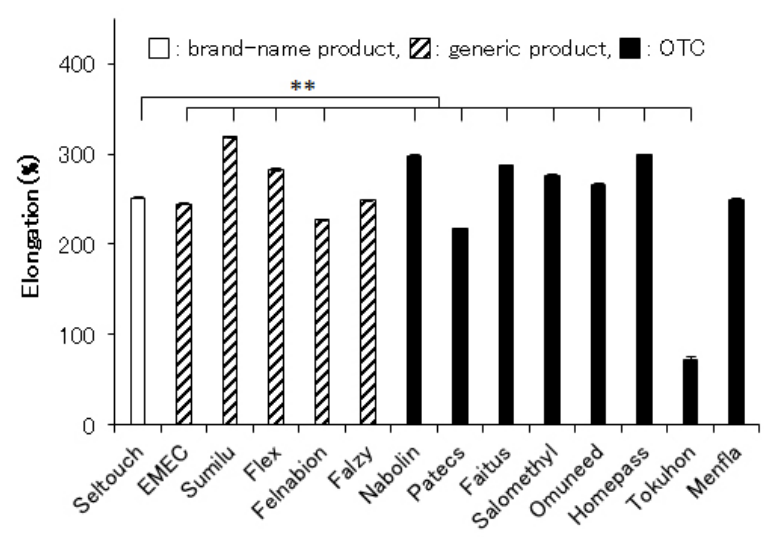

Figure 5. Comparison of elongation of the various products $(\boldsymbol{n}=\mathbf{3}) .{ }^{* *} p<0.01$ (brand-name $v s$. generics, Dunnett's test). 


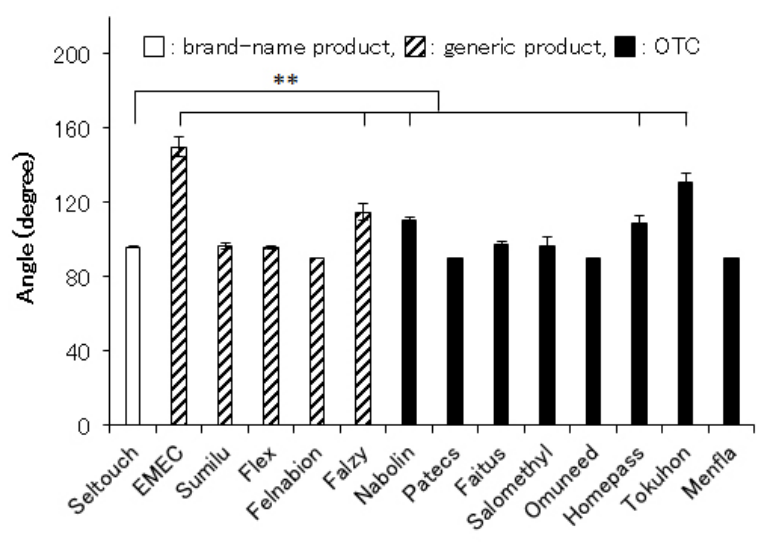

Figure 6. Comparison of flexibility of the support of the various products $(\boldsymbol{n}=\mathbf{3}) .{ }^{* *} p<0.01$ (brand-name $v s$. generics, Dunnett's test).

Tokuhon $(72 \%)$ was lower than that of Seltouch. In addition, significance tests of various products were conducted. There were significant differences between Falzy/OTC products other than Menfla and Seltouch $(p<$ 0.01) (Figure 5).

\subsection{Measurement of the flexibility of the support layer}

When the support layer of each preparation is highly flexible, a portion of the preparation often adheres to another portion on removing a liner. However, this can be prevented if the rigidity of the support layer is adequate. The results of support-layer flexibility measurement are shown in Figure 6. The flexibilities of EMEC (150 degrees), Falzy (115 degrees), Nabolin (110 degrees), Homepass (108 degrees), and Tokuhon (131 degrees) were higher than that of Seltouch (96 degrees). In addition, significance tests of these products were conducted. EMEC, Falzy, Nabolin, Homepass, and Tokuhon showed significant differences in comparison with Seltouch $(p<0.01)$ (Figure 6).

\subsection{Measurement of the interval until exfoliation from artificial skin in a wet state}

Some tape preparations attached to the skin may easily peel off in a wet state. We examined the interval until various preparations peel from artificial skin in a wet state, as shown in Figure 7. The intervals of EMEC (1.4 $\mathrm{min})$, Flex (30.5 min), Falzy (24.1 min), Nabolin (0.2 $\mathrm{min}$ ), Patecs (26.3 $\mathrm{min}$ ), and Homepass (15.6 min) were shorter than that of Seltouch (120 min). In addition, significance tests of these products were conducted. EMEC, Flex, Falzy, Nabolin, Patecs, and Homepass showed significant differences in comparison with Seltouch $(p<0.01)$ (Figure 7).

\section{Discussion}

The $\mathrm{pH}$ of each preparation may influence its stability

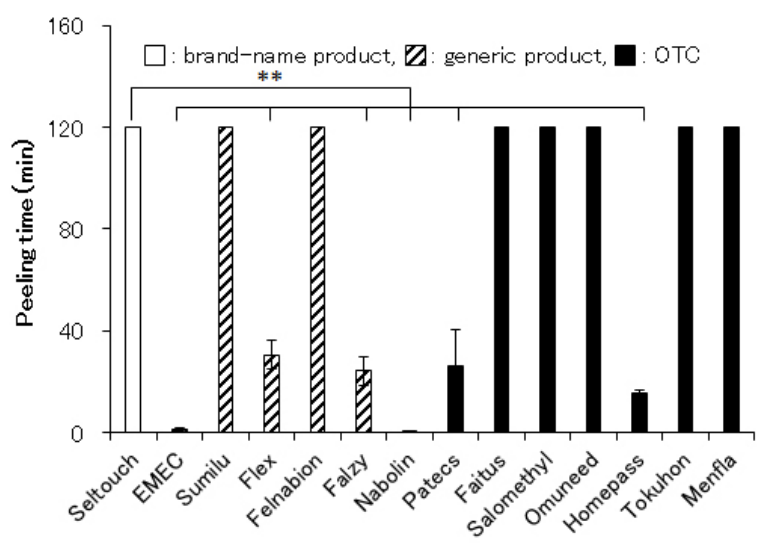

Figure 7. Comparison of the peeling time of the various products $(\boldsymbol{n}=\mathbf{3}) .{ }^{*} * p<0.01$ (brand-name $v s$. generics, Dunnett's test).

and comfortableness, such as stimulation at the time of use. The skin surface $\mathrm{pH}$ is 4 to 5.5 (weakly acidic) in healthy adults (14). However, according to a study, the skin may be readily influenced by external stimuli when the $\mathrm{pH}$ of a preparation is lower than that of the skin surface, or when it is alkaline; the skin may become more sensitive (15). We measured the $\mathrm{pH}$ of each preparation. The $\mathrm{pH}$ values of most preparations used in this study were approximately 5 , being within the skin $\mathrm{pH}$ range of healthy adults (Figure 1). On the other hand, the $\mathrm{pH}$ values of EMEC and Tokuhon were 7.0 , being higher than that of the skin surface in healthy adults $(\mathrm{pH} 4$. to 6.0). However, the $\mathrm{pH}$ values of these preparations were within the weakly acidic to neutral range; and therefore, there may be no harmful effects on the skin.

Skin stimuli related to patch preparations are primarily classified into 3 types: physical stimuli associated with strong adhesive force related corneal layer exfoliation/injury or support layer stress-related dermal stress, chemical stimuli related to the transfer of an active ingredient from the skin surface to the inner area, and maceration stimuli associated with the absence of water-vapor permeability on sweating (10). Felbinaccontaining tape preparations are anti-inflammatory analgesic drugs, and may be used at the affected site for a long period, suggesting the marked influence of maceration stimuli. We examined the presence or absence of maceration stimuli by measuring the water evaporation (water-vapor permeability) from each preparation (Figure 2). The water-vapor permeabilities of the tape preparations used in this study ranged from 169 to $380 \mathrm{~g} / \mathrm{m}^{2} / 24 \mathrm{~h}$. In particular, EMEC showed the highest value $\left(380 \mathrm{~g} / \mathrm{m}^{2} / 24 \mathrm{~h}\right)$. This suggests that EMEC with a high water-vapor permeability does not frequently induce maceration stimuli. However, an increase in the water-vapor permeability reduces the sealing property of a patch preparation, decreasing the skin transfer of the drug; and therefore caution is needed. Therefore, it may be necessary to select drugs 
in accordance with patients' wishes (10).

Preparations with a strong adhesive force may not peel off when attached to the skin, whereas those with a weak adhesive force tend to peel off. In particular, preparations with a weak adhesive force may be turned off or peel off due to clothes-related friction after attachment or attachment to mobile areas, such as the elbows and knees, directly influencing patients' comfortableness $(4,8)$. Using the inclined ball tack test, we measured the adhesive forces of various preparations based on the ball weight (Figure 3). The adhesive forces of Salomethyl, Homepass, and Tokuhon (1.04 g, highest) were higher than that of Seltouch $(0.06 \mathrm{~g})$. These results showed that the adhesive forces of all other preparations were higher than that of Seltouch, suggesting that they do not peel off.

The peeling-force refers to the force required to peel off the preparation. Tape preparations with a strong adhesive force are attachable to the skin even in mobile areas, such as the elbows and knees, facilitating attachment for a long period. However, the strong adhesive force may cause physical stimuli, such as corneal-layer exfoliation on peeling, inducing symptoms, such as flare (16). We evaluated the peelingforces of various preparations by measuring the force required to peel off the preparation $(\mathrm{N})$ (Figure 4). The values ranged from 0.4 to $4.9 \mathrm{~N}$. The peeling-force of Omuneed was the highest $(4.9 \mathrm{~N})$, followed by that of Falzy $(4.1 \mathrm{~N})$. These results show that a peelingforce 4 to 5 times higher than that of Seltouch $(0.9 \mathrm{~N})$ was required to peel off these preparations, suggesting that it induces physical stimuli, such as corneal-layer exfoliation, after peeling.

Many tape preparations containing felbinac are available for articular areas, such as the knees and elbows, due to their support flexibility. Non-flexible preparations may not resist affected-site motions when attached to a mobile region, leading to turning-up or peeling. Therefore, it is important to adopt a support layer consisting of a flexible material. Furthermore, flexible preparations are more closely adhered to the skin, increasing the permeability of an active ingredient; and they may be more effective (13). We measured the elongation rate $(\%)$ in comparison with the baseline length by elongating various preparations at a specific force (Figure 5). The elongation rates of most preparations ranged from 217 to $319 \%$. That of Sumilu was the highest (319\%), followed by those of Homepass (299\%) and Nabolin (298\%). On the other hand, the elongation rate of Tokuhon was low (72\%). These results suggest that the preparations, excluding Tokuhon, are extensible (elongation rate: $\geq 200 \%$ ).

When attaching a preparation to the skin, it is possible to effectively permeate an active ingredient by removing the liner, as indicated in the package inserts, and attaching the preparation to the affected site, although methods differ among various preparations.
However, concerning attachment to the back, lumbar, and gluteal region, a portion of the preparation often adheres to another portion on removing the liner. This may occur when the support layer of a preparation is flexible. When the support layer is adequately hard, this can be prevented. We measured the angle of each preparation's flexion to investigate the flexibility of the support layers of various preparations, and considered that preparations with a greater angle were more flexible (Figure 6). Most tape preparations containing felbinac used in this study, including Seltouch (96 degrees), showed an angle of 90 to 115 degrees. However, EMEC and Tokuhon showed higher values (150 and 131 degrees, respectively). This suggests that the support layers of these products are more flexible than those of the other products, leading to adhesion between a portion and another portion or wrinkles on attachment. On the other hand, hard preparations with a less flexible support layer are not attachable to mobile areas, such as joints, which may result in patients' discomfort (13). Therefore, the flexibility of the support layer must be considered for drug selection.

A patch preparation can be readily peeled from the affected site by getting it wet with water or warm water. However, a patch preparation that does not readily peel off is more appropriate for some sites to become wet, such as the wrists. We examined the interval until exfoliation from artificial skin in a wet state. The values of EMEC and Nabolin were 1.4 and $0.2 \mathrm{~min}$, respectively. Furthermore, those of Flex and Homepass were 30.5 and $15.6 \mathrm{~min}$, respectively (Figure 7). On the other hand, 8 products (Sumilu, Felnabion, Faitus, Salomethyl, Omuneed, Tokuhon, Menfla, and Seltouch) did not peel off even after $120 \mathrm{~min}$, suggesting their water resistance.

The results of this study showed that there were differences in pharmaceutical properties among the tape preparations containing the same component. These properties may influence patients' comfortableness. If the properties of various preparations are understood, their requests may be met. For example, when patients attach one of the felbinac-containing tape preparations used in this study complain of stuffiness, a product with a higher water-vapor permeability can be recommended. When patients complain of pain on exfoliation, a product with a smaller peeling-force can be recommended. When patients complain of a short interval until exfoliation, a product with a stronger adhesive force should be selected. For patients attaching a product to an articular site, a product with a higher elongation rate, that is, a stretchable product, can be recommended. For patients with impaired hands or those living alone, a product that can be attached by themselves (a product with a hard support layer preventing adhesion to fingers or portion-to-portion adhesion) should be selected. In addition, a waterproof product should be selected for housewives, and a low-adhesiveness product should 
be recommended for patients who wish to attach a tape preparation that can be readily peeled off in a wet state. Thus, pharmacists can understand these properties based on the results of this study, and select preparations appropriate for individual patients, leading to more favorable drug adherence.

\section{References}

1. Seltouch ${ }^{\circledR}$ Tape 70, Pharmaceutical products interview form, Pfizer Japan Inc. October, 2014 (ten revision). (in Japanese)

2. Ministry of Health, Labour and Welfare. Trend of recent medical expenses, [approximate medical expenses], February, 2015 issue. http://www.mhlw.go.jp/topics/ medias/month/15/02.html (accessed September 18, 2016). (in Japanese)

3. Ohtanti M, Matsumoto M, Namiki M, Yamamura Y, Sugiura M, Uchino K. Evaluation of pharmaceutical equivalency between genuine and generic ketoprofen tape. J Pharm Sci Tech Jpn. 2011; 71:120-125. (in Japanese)

4. Takuya Uematsu, Atsushi Kobayashi, Kenichi Serizawa, Fumikazu Kinoshita, Toru Matsuura, Akihito Nagumo, Tomoyuki Kakinuma, Takahiro Sugao. Comparison of adhesive force between brand-name and generic ketoprofen tapes with tack rolling ball test. J Jpn Soc Hosp Pharm. 2015; 52:513-517. (in Japanese)

5. Wada Y, Nozawa M, Goto M, Shimokawa K, Ishii F. Generic selection criteria for safety and patient benefit [I]: Comparing the original drugs and generic ones in pharmaceutical properties. J Community Pharm Pharm Sci. 2014; 6:97-105. (in Japanese)

6. Nozawa M, Wada Y, Yamazaki N, Shimokawa K, Ishii F. Generic selection criteria for safety and patient benefit [II]: Physicochemical characteristics of original versus generic drugs for different difluprednate-containing products (ointment, cream, and lotion). Jpn Soc Community Pharm Pharm. 2014; 2:37-48. (in Japanese)

7. Wada Y, Nozawa M, Goto M, Shimokawa K, Ishii F. Generic selection criteria for safety and patient benefit [III]: Comparing the pharmaceutical properties and patient usability of original and generic ophthalmic solutions containing Timolol maleate. Jpn Soc Pharm Health Care Sci. 2015; 41:394-403. (in Japanese)

8. Wada Y, Kihara M, Nozawa M, Shimokawa K, Ishii F. Generic selection criteria for safety and patient benefit [IV]: Physicochemical and pharmaceutical properties of brand-name and generic ketoprofen tapes. Drug Discov Ther. 2015; 9:229-233.

9. Wada Y, Ami S, Nozawa M, Goto M, Shimokawa K, Ishii F. Generic selection criteria for safety and patient benefit [V]: Comparing the pharmaceutical properties and patient usability of original and generic nasal spray containing ketotifen fumarate. Drug Discov Ther. 2016; 10:88-92.

10. Hiyoshi M. Pharmaceutical assessment of Romal ${ }^{\circledR}$ tape 20/40 in ketoprofen tape. Prog Med. 2009; 29:193-196. (In Japanese)

11. JIS Z 0237: 2009 Testing methods of pressure-sensitive adhesive tapes and sheets. http://kikakurui.com/z0/ Z0237-2009-01.html (accessed September 18, 2016). (in Japanese)

12. Miura T, Matsuzaki H, Nouno H. Questions from patients: Adhesiveness of percutaneous bronchodilator delivery system tulobuterol. J Ambul Gen Ped. 2008; 11:14-19. (in Japanese)

13. Terada H. Pharmaceutical characteristics and pharmacologic effect of NSAID pap. Jpn J Med Pharm Sci. 2007; 58:49-54. (in Japanese)

14. Kuriyama S, Tomonari H, Hayashi F, Numata M, Kimura H, Kanome K, Ebihara T, Kawaguchi Y, Hosoya T. The role of the skin $\mathrm{pH}$-acid mantle in exit-site infection in CARD patients. Nihon Toseki Igakkai Zasshi. 1998; 31:997-1000. (in Japanese)

15. Ueno M, Kanda K, Kasagi N. Effect of tape stripping and skin cleansing repeatedly on skin barrier. J Yonago Med Ass. 2006; 57:103-112. (in Japanese)

16. Shinkai N, Okumura Y, Saito H, Kusunoki A, Yamauchi H. Drug properties and skin irritation of antiinflammatory analgesic agent. Pharma Medica. 2007; 25:113-117. (in Japanese)

(Received August 23, 2016; Revised September 19, 2016; Accepted September 20, 2016) 